\title{
Correction to: Myths of the Creation of Chinese
}

\section{Correction to:}

\section{Z. Tian et al., Myths of the Creation of Chinese, https://doi.org/10.1007/978-981-15-5928-0}

The original version of the book was inadvertently published with a few errors. The book has also been updated with these corrections.

1. Change of author's affiliation for Shuxian Ye, changed "Zhiyuan College" to "Center for Study of Literary Anthropology".

2. The textual corrections have been carried out throughout the book. 